
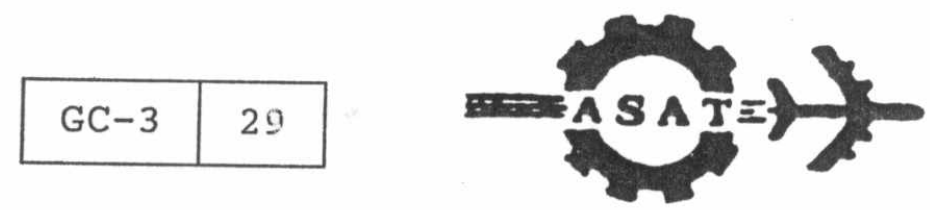

$\Gamma$

\title{
TOWARDS PERFORMANCE IMPROVEMENT FOR RADIO FUZES OF AA/C GUIDED MISSILES
}

M.T.C , Cairo

\section{ABSTRACT}

The fuze is the equipment of the missile which is responsible for initiating the warhead (W.H) explosion. The performance of the fuze or its efficiency is measured by the probability of target destruction which it can achieve. To improve a fuze performance means to increase the probability of target destruction by that fuze. This may be realized by increasing the degree of identity between the fuze activation zone (F.A.Z) and the target destruction zone (T.D.Z). The matching process may be either an actual one by using radio fuze(R.F) with variable antenna beam pattern(A.B.P) or virtual one by delaying the W.H initiation. This delay is calculated according to the real missile-target $(\mathrm{M} / \mathrm{T})$ encounter conditions.

The present paper deals with the analysis of methods for R.F performance improvement in the cases of R.F with variable A.B.P and that with fixed A.B.P. The paper provides a mathematical model which is used to determine the relative velocity modulus necessary for determination of the W.H initiation optimum time instant. In the case of R.F with variable A.B.P, the model determines the optimum tilting angle of the antenna beam with respect to the missile axis. For R.F with fixed A.B.P, the model determines the corresponding delay time that do matching between the F.A.Z and the T.D.Z for a specific encounter conditions.

The improved performance is calculated for some specific encounter conditions and the results are evaluated. A performance improvement factor (P.I.F) is defined which value may reach $83.7 \%$.

\footnotetext{
* M. EL-LITHY : Ass. Prof. - Guidance Department, M.T.C, Cairo

* A. A. SALEH : M. Sc - M.T.C, Cairo 


\section{1- INTRODUCTION}

The fuzing system in $\mathrm{AA} / \mathrm{C}$ guided missile aims to increase the probability of target destruction through a proper initiation of the W.H. Information about the missile velocity, target velocity and the encounter angle are necessary for precise determination of encounter zone (E.Z) . The fuze performance is quantitatively defined by the ratio of actual vulnerable target area located in the T.D.Z at the moment of W.H initiation, to the maximum vulnerable target area[1]. Improvement of fuze performance is always aiming at the maximum coincidence between the F.A.Z and the T.D.Z by the use of the above information as well as the W.H parameters (W.H static destruction angle $\alpha_{\mathrm{st}}$ and fragment speed $\left.\mathrm{Vd}[1]\right)$.

\section{2- IMPROVEMENT OF RADIO FUZE PERFORMANCE}

It is the same thing to match the F.A.Z to the T.D.Z or vice versa. The matching process is accomplished through either one of the following approaches :

A- Using of variable activation zone which axis is adjusted with respect to the missile axis according to the encounter conditions. Such variation may be :

1- Variable antenna beam pattern which may take place continuously or at least in steps.

2- Variable delay time R.F, where the initiation of the W.H will be delayed according to the real encounter conditions.

B- Using of W.H where the destruction zone orientation in the space is adjustable. This orientation may be forward or backward. It can be realized through one of the following ways :

1- The inclination of the T.D.Z with respect to the missile axis may be varied through variation of the pyrotechnique exploder position on the W.H detonation tube which has to be selected according to the real encounter conditions.

2- The variation of T.D.Z orientation in the space may be realized by rotating the W.H around an axis perpendicular to the missile axis.

In the following analysis, attention will be paid to the R.F with variable activation zone.

\section{1- Radio Fuze with Variable Antenna Beam Pattern}

The R.F with variable A.B.P uses the tilting beam angle as a variable parameter to maximize the degree of identity between F.A.Z and T.D.Z according to information about either missile speed, relative velocity (R.V) modulus or R.V vector. In the following analysis of performance improvement, the algorithm of R.F performance calculation[2] is used with a slight modification to consider only a part of the E.Z which will be determined according to the available information controlling the tilting beam angle.

\subsection{1- Improvement of Radio Fuze Performance according to information about the Missile Speed}

Information about missile speed results in an appreciable shrinkage of the E.Z. This situation is demonstrated in figure 1, where two E.Zs are depicted. The first one, represented by the hatched area, is corresponding to a known value of missile speed. The other one, represented by the whole 
area, is corresponding to a certain range within which the missile speed varies. This shrinkage of the E.Z means that the R.V between the missile and target varies within a close range, thus increasing the matching between the F.A.Z and the T.D.Z and consequently the fuze performance.

The performance of R.F based on information about 'missile speed is calculated for the missile speeds $600 \mathrm{~m} / \mathrm{s}, 900 \mathrm{~m} / \mathrm{s}$ and $1200 \mathrm{~m} / \mathrm{s}$. The performance was found to be $54 \%, 53.3 \%$ and $52.8 \%$ at the beam tilting angle of $73 \mathrm{deg}$, $67 \mathrm{deg}$. and $62 \mathrm{deg}$. respectively.

\subsection{2- Improvement of Radio Fuze Performance according to information about the Relative Velocity Modulus}

The F.A.Z varies depending on the value of the R.V modulus. For a given value of relative speed the E.Z tends to be a curve. This situation is demonstrated in figure 2, the E.Z corresponding to information about the missile speed is seen by the angle $\psi 1$ from the missile center, while the E.Z (represented by a curve) corresponding to information about the R.V modulus is seen by the angle $\psi 2$ (which is smaller than $\psi 1$ ). Therefore, information about the missile speed leads to a more shrinkage of the E.Z and consequently a better matching between the F.A.Z and the T.D.Z.

The performance of R.F based on information about relative speed is calculated for the relative speeds $900 \mathrm{~m} / \mathrm{s}, 1200 \mathrm{~m} / \mathrm{s}$ and $1500 \mathrm{~m} / \mathrm{s}$. The performance was found to be $79 \%, 73.8 \%$ and $68 \%$ at the beam tilting angle of $75 \mathrm{deg}$. , $70 \mathrm{deg}$. and $64 \mathrm{deg}$. respectively.

\subsection{3- Improvement of Radio Fuze Performance according to information about Relative Velocity Vector}

A complete information about the R.V vector reduces the E.Z to a point and the fuze performance is maximized. For a given value of R.V, the W.H is activated when the antenna beam tilting angle equals $\phi_{S}$ as shown in figure 3 , where $\phi_{s}$ is given by :

$$
\phi_{\mathrm{s}}=\tan ^{-1}\left(\frac{\mathrm{V}_{\mathrm{d}}-\left|\overline{\mathrm{V}}_{\mathrm{r}}\right| \cos \theta}{\left|\overline{\mathrm{V}}_{\mathrm{r}}\right| \sin \theta}\right)
$$

\section{2-Radio Fuze with Variable Delay Time}

In this type of fuze, the F.A.Z is matched to the T.D.Z through a delay time, the value of which is determined according to the real encounter conditions. The R.F with variable delay time uses, in general, uncontrolled A.B.P that makes an angle $\phi \mathrm{ps}$ (preliminary triggering angle ) with the missile axis. This angle specifies the antenna beam tilting angle corresponding to the minimum allowable delay time for reliable M/T collision. 
As soon as the target has entered the A.B.P (theoretically when the target gravity center crosses the direction of main maximum)a preliminary fuze triggering takes place (point $\mathrm{A}$ in figure 3). The W.H activation occurs after a definite delay time $\tau$ where the target crosses the destruction zone axis (point B).

The delay time is controlled by the distance $\mathrm{D}$ (from point $\mathrm{A}$ to point $\mathrm{B}$ ) and the R.V (Vr) between target and missile. The distance $\mathrm{D}$ itself depends on the fragment speed (Vd), the $\mathrm{M} / \mathrm{T}$ miss distance $(\rho)$, and the antenna beam tilting angle $\left(\phi_{\mathrm{ps}}\right)$.

The relation $\tau=f\left(V_{r}, V_{d}, \rho, \phi_{p s}\right)$ is given by [2] :

$$
\tau=\frac{\rho}{\left|\bar{V}_{r}\right|} \sqrt{1+\beta^{2}\left(1+m_{2}^{2}\right)+2 \beta\left(\cos \phi_{p s}+m_{2} \sin \phi_{p s}\right)}
$$

Where

$$
\begin{aligned}
& m_{1}=\tan (90+\theta) \\
& m_{2}=\frac{v_{d}-\left|\overline{v_{r}}\right| \cos \theta}{\left|\overline{v_{r}}\right| \sin \theta} \\
& \beta=\frac{\sin \phi_{p s}-m_{1} \cos \phi_{p s}}{m_{1}-m_{2}} \\
& \theta \ldots \text { is the angle that the R.V makes with the negative Y-axis }
\end{aligned}
$$

If the target velocity is parallel with the missile velocity $(\theta=90 \mathrm{deg}$.) the above equation is reduced to :

$$
\tau=\frac{\rho}{\left|\bar{V}_{r}\right| V_{d}}\left(V_{d} \cos \phi_{p s}-\left|\bar{V}_{r}\right| \sin \phi_{p s}\right)
$$

The algorithm and the flowchart for performance evaluation of such R.F are detailed in [2].

The performance of R.F based on variable delay time is calculated using the mentioned algorithm for the following parameters value :

- Fragment speed $\quad 3000 \mathrm{~m} / \mathrm{s}$

- Missile speed range $550-1100 \mathrm{~m} / \mathrm{s}$

- Encounter angle

$120 \mathrm{deg}$

- M/T miss-distance $60 \mathrm{~m}$
- W.H static destruction angle $10 \mathrm{deg}$ - Target speed range $\quad 350-700 \mathrm{~m} / \mathrm{s}$

- Target equivalent length $\quad 10 \mathrm{~m}$

- Beam tilting angle $20 \mathrm{deg}$

The dependence of R.F performance on delay time is shown in figure 4 . The maximum performance was found to be $17.5 \%$ at a delay time of $38 \mathrm{~m} \mathrm{sec}$. It was found that the performance of R.F based on variable delay time is poor compared to R.F with variable antenna beam. 


\subsection{1- Improvement of Radio Fuze Performance according to information about Missile Speed}

The expected shrinkage of the E.Z due to information about missile speed results in a higher fuze performance. The performance of R.F with variable delay time based on information about missile speed is calculated for the missile speed of $600 \mathrm{~m} / \mathrm{s}, 900 \mathrm{~m} / \mathrm{s}$, and $1200 \mathrm{~m} / \mathrm{s}$. The R.F performance was found to be $30.8 \%, 34.4 \%$, and $36 \%$ at a delay time of $24 \mathrm{~m} \mathrm{sec}, 32 \mathrm{~m}$ $\mathrm{sec}$, and $24 \mathrm{~m} \mathrm{sec}$ respectively.

\subsection{2- Improvement of Radio Fuze performance according to information about Relative Velocity Modulus}

The performance of R.F with variable delay time based on information about R.V modulus is calculated for the relative speed of $900 \mathrm{~m} / \mathrm{s}, 1100 \mathrm{~m} / \mathrm{s}$, and $1300 \mathrm{~m} / \mathrm{s}$. The R.F performance was found to be $56.4 \%, 54.7 \%$, and $51.3 \%$ at a delay time of $56 \mathrm{~m} \mathrm{sec}, 42 \mathrm{~m}$ $\mathrm{sec}$, and $32 \mathrm{~m} \mathrm{sec}$ respectively.

\section{3- METHODOLOGY OF PERFORMANCE IMPROVEMENT}

In the following analysis, a mathematical model is elaborated which provides an information about the $\mathrm{M} / \mathrm{T}$ relative velocity. Such information aids the matching process between the F.A.Z and the T.D.Z .

\section{1- Radio Fuze with Variable Antenna Beam Pattern}

The M/T miss distance $(\rho)$ and beam tilting angle $(\phi)$ are assumed to be periodically measured, their rates of change $(\dot{\rho}$ and $\dot{\phi})$ are calculated. The F.A.Z and T.D.Z are determined from the measured and calculated $\rho, \phi, \dot{\rho}$ and $\dot{\phi}$.

Consider the coordinate system $(\rho, \theta, \phi)$ shown in figure 5 , the $\mathrm{M} / \mathrm{T}$ relative motion is described in the plane of fire (the plane contains the missile longitudinal axis X1-axis, and the target theoretical center). The value of $\theta$ is not important, since the fragments are uniformly distributed in $\mathrm{Y}-\mathrm{Z}$ plane, extended to certain angle $\alpha_{s t}$ in $\mathrm{X}-\mathrm{Y}$ plane as shown in figure 6 . The components of the M/T miss-distance $\rho$ in both the missile longitudinal axis (X1) and line of intersection of the plane of fire with the $\mathrm{Y}-\mathrm{Z}$ plane are :

$$
\begin{aligned}
& \rho_{\mathrm{x} 1}=\rho \cos \phi \\
& \rho_{\mathrm{yz}}=\rho \sin \phi
\end{aligned}
$$


The rates of change of $\rho_{\mathrm{x} \text { I }}$ and $\rho_{\mathrm{Yz}}$ are :

$$
\begin{aligned}
& V_{\rho_{X 1}}=\frac{d \rho_{X 1}}{d t}=\dot{\rho} \cos \phi-\rho \dot{\phi} \sin \phi \\
& V_{\rho_{r z}}=\frac{d \rho_{Y Z}}{d t}=\dot{\rho} \sin \phi+\rho \dot{\phi} \cos \phi
\end{aligned}
$$

In other words, the $M / T$ relative motion is described by the distances $\rho_{X 1}$ on $X 1$-axis, $\rho_{Y Z}$ in the W-plane, and their rates of change $\left(\mathrm{v}_{\rho_{\mathrm{x} 1}}, \mathrm{v}_{\rho_{\mathrm{yz}}}\right)$ as shown in figure 7 . Now, based on the above description of the $\mathrm{M} / \mathrm{T}$ relative motion, the angle $\left(\phi_{\mathrm{s}}\right)$ that the central axis of T.D.Z makes with the $\mathrm{X} 1$-axis is given by :

$$
\phi_{\mathrm{s}}=\tan ^{-1}\left(\frac{\mathrm{v}_{\mathrm{d}}}{\mathrm{v}_{\rho_{\mathrm{x} 1}}}\right)
$$

Where

$$
\mathrm{V}_{d} \cdots \text { is the fragment speed }
$$

As the target is being continuously tracked, the W.H is activated when the antenna beam tilting angle coincides with the angle $\phi_{\mathrm{s}}$. Due to the time of fuze inertia $\left(t_{\text {in }}\right)$, the beam tilting angle at which the W.H is activated $\left(\phi_{\text {in }}\right)$ may be calculated as follows :

From successive measurement of target range $\rho$ and beam tilting angle $\phi$, the velocities $\mathrm{V}_{\rho_{\mathrm{x}}}$ and $\mathrm{V}_{\rho_{\mathrm{yz}}}$ are calculated. At any instant, the time $\tau_{\mathrm{i}}$ at which the target crosses the central axis of target destruction zone, assuming constant $\mathrm{M} / \mathrm{T}$ relative velocity as shown in figure 8 , is given by :

$$
\tau_{i}=\frac{D_{i}}{V_{r}}
$$

Where

$$
\begin{aligned}
V_{r} & =\sqrt{V_{r_{x 1}}^{2}+V_{r_{z z}}^{2}} \\
D_{i} & =\sqrt{\left(\rho \cos \phi-X_{i}\right)^{2}+\left(\rho \sin \phi-Y_{i}\right)^{2}} \\
X_{i} & =\frac{\rho(\sin \phi-m 1 \cos \phi)}{m 2-m 1} \\
Y_{i} & =m 2^{*} X_{i} \\
m 1 & =\frac{V_{\rho_{r z}}}{V_{\rho_{x 1}}} \\
m 2 & =\frac{V_{d}}{V_{\rho_{x 1}}}
\end{aligned}
$$


Then, the angle $\left(\phi_{\text {in }}\right)$ is given by :

$$
\phi_{i n}=\tan ^{-1}\left[\frac{\rho \sin \phi-V_{r_{1 z}}\left(\tau_{i}-t_{i n}\right)}{\rho \cos \phi-V_{r_{x 1}}\left(\tau_{i}-t_{i n}\right)}\right]
$$

On board of the missile, the differentiation of $\rho$ and $\phi$ is obtained by means of approximate differentiation. The following formula is based on Newton's second interpolation formula up to fifth order of finite difference for the case that the function is specified at equally spaced points [3] :

$$
P_{n}^{\prime}=\sum_{i=0}^{5} \alpha_{5-i} * y_{i}
$$

where

$$
\begin{array}{lll}
\alpha_{0}=\frac{137}{60 h} & \alpha_{1}=-\frac{5}{h} & \alpha_{2}=\frac{5}{h} \\
\alpha_{3}=-\frac{10}{3 h} & \alpha_{4}=\frac{5}{4 h} & \alpha_{5}=-\frac{1}{5 h}
\end{array}
$$

The parameter $\mathrm{h}$ is the time difference between two successive measurements of $\rho$ and $\phi$.In practice, $\mathrm{h}$ is equal to pulse repetition period for pulse radar or sampling rate for FMCW radar. At any time the value of $\dot{\rho}$ (similarly is $\dot{\phi}$ ) is given by :

$$
\dot{\rho}_{k}(t)=\left[\alpha_{0}\left|\alpha_{1}\right| \alpha_{2}\left|\alpha_{3}\right| \alpha_{4} \mid \alpha_{5}\left[\begin{array}{l}
\frac{\frac{\rho_{k}(t)}{\rho_{k-1}(t)}}{\frac{\rho_{k-2}(t)}{\rho_{k-3}(t)}} \\
\frac{\rho_{k-4}(t)}{\rho_{k-5}(t)}
\end{array}\right]\right.
$$

As the value of $\rho$ changes with time the value of $\dot{\rho}$ is periodically calculated according to the following matrix formula :

$$
\left[\begin{array}{c}
\rho_{k}(t+T) \\
\rho_{K-1}(t+T) \\
\rho_{K-2}(t+T) \\
\rho_{K-3}(t+T) \\
\rho_{K-4}(t+T) \\
\rho_{K-5}(t+T)
\end{array}\right]=\left[\begin{array}{llllll}
0 & 0 & 0 & 0 & 0 & 0 \\
0 & 1 & 0 & 0 & 0 & 0 \\
0 & 0 & 1 & 0 & 0 & 0 \\
0 & 0 & 0 & 1 & 0 & 0 \\
0 & 0 & 0 & 0 & 1 & 0 \\
0 & 0 & 0 & 0 & 0 & 1
\end{array}\right]\left[\begin{array}{c}
0 \\
\rho_{k}(t) \\
\rho_{K-1}(t) \\
\rho_{K-2}(t) \\
\rho_{K-3}(t) \\
\rho_{K-4}(t)
\end{array}\right]+\left[\begin{array}{c}
1 \\
0 \\
0 \\
0 \\
0 \\
0
\end{array}\right] \rho_{K}(t+T)
$$

where

$\rho_{\mathrm{K}-\mathrm{n}} \ldots$ The $\mathrm{n}^{\text {th }}$ delay of the measured target miss-distance

$\mathrm{T} \ldots$. . The time period between two successive measurements of $\mathrm{M} / \mathrm{T}$ miss-distance 
In conclusion, the target range and beam tilting angle are periodically measured, their rates of change are periodically calculated. At each instant the time $\left(\tau_{i}\right)$ and the angle $\left(\phi_{i n}\right)$ are calculated. The W.H will be initiated when either the time $\left(\tau_{i}\right)$ is equal to the time of fuze inertia $\left(t_{\text {in }}\right)$ or the beam tilting angle is equal to the angle $\left(\phi_{\text {in }}\right)$.

\section{2- Radio Fuze with Fixed Antenna Beam}

For R.F with fixed antenna beam, the target will cross the A.B.P in a definite time through which an information about $\mathrm{M} / \mathrm{T}$ relative velocity is calculated and used for evaluating a delay time to realize matching between the F.A.Z and the T.D.Z . In the following approach, the phenomenon of Doppler effect is used to determine a limited range within which the $M / T$ relative velocity varies. An average value for speed and direction of $\mathrm{M} / \mathrm{T}$ relative velocity is calculated from the obtained range limits as follows :

A R.V vector (Vr), see figure 9, is decomposed into a radial component (Vrr) on the line joining the missile and target, and a normal component ( Vrn $)$ in the direction perpendicular to Vrr in the plane containing the target velocity and the missile velocity. It is known that the frequency shift (fd) in the transmitted frequency due to the relative motion between the target and missile is given by [4] :

Where

$$
\mathrm{f}_{d}= \pm \frac{2 \mathrm{~V}_{\mathrm{r}} \cos \gamma}{\lambda}
$$

$V_{r} \ldots$ R.V between radar and the target

$\lambda \quad \ldots$ wavelength of the transmitted wave.

$\gamma \quad \ldots$ angle between the R.V vector and line joining the missile and target.

The relation between the radial component of R.V $\left(v_{r r}\right)$ and the frequency shift $\left(f_{d}\right)$ is given by :

$$
V_{r r}=V_{r} \cos \gamma=\frac{\lambda}{2} f_{d}
$$

The target rate of approach along the missile longitudinal axis (x1-axis ) is given by :

Where

$$
V_{r x}=V_{r r x}+V_{r n x}
$$

$\left(V_{r x}\right) \ldots X \mathrm{X} 1$ - component of R.V vector .

$\left(V_{r r x}\right) \ldots X 1$ - component of the radial component .

$\left(V_{r n x}\right) \ldots X 1$ - component of the normal component. 
The value of Vrrx is determined if the Doppler frequency is known, as shown from the following equation :

$$
V_{r r x}=V_{r r} \cos \phi=\frac{\lambda}{2} f_{d} \cos \phi
$$

On the other hand, the normal component of the R.V and consequently its projection on $\mathrm{x} 1$-axis are unknown, therefore the radial component offers no valuable information on the R.V vector $\left(v_{r}\right)$, and its direction could vary between $\gamma=0^{\circ}$ and $\gamma \cong 90^{\circ}$ passing over or under the missile.

Up till now, the destruction zone central axis can't be determined, even roughly, and the following analysis aims to decrease the range within which the angle $\gamma$ varies, using the information about target speed range and missile speed .

Assume that the maximum and minimum expected target speeds are $V_{t_{\max }}, V_{t_{\min }}$ respectively, and the maximum angle of attack that the approaching target makes with the missile longitudinal axis is $\eta \quad$ (as shown in figure 10), then the maximum and minimum values of the radial component of the R.V are calculated as follows:

$$
w=\tan ^{-1}\left(\frac{V_{t \min } \sin \eta}{V_{m}+V_{t \min } \cos \eta}\right)
$$

Where

$w \ldots$ the angle that the minimum R.V makes with the missile longitudinal axis. $v_{m} \ldots$ missile speed.

The minimum value of the R.V vector is :

$$
V_{r \min }=\sqrt{\left(V_{t \min } \sin \eta\right)^{2}+\left(V_{m}+V_{t \min } \cos \eta\right)^{2}}
$$

The minimum value of the radial component $\left(V_{r r \min }\right)$ the R.V vector is:

$$
V_{r r \min }=V_{r \min } \cos (W+\phi)
$$

The maximum value of the radial component, $\left(V_{r r \max }\right)$ of the R.V is taken as :

$$
V_{r r \max }=V_{m}+V_{t \max }
$$


Assume that $\mathrm{V}_{\pi}$ is the measured value of radial component of the R.V vector $V_{r}$, then the maximum and minimum value of angle $\gamma$ could be determined, as shown in figure 11 , from the following equation :

$$
\begin{aligned}
& V_{r r}=\frac{\lambda}{2} f_{d}=V_{r \text { max }} \cos \gamma_{\text {max }} \\
& V_{r r}=\frac{\lambda}{2} f_{d}=V_{r \text { min }} \cos \gamma_{\text {min }} \\
& \gamma_{\text {max }}=\cos ^{-1}\left(\frac{V_{r r}}{\left.V_{r \text { max }}\right)}\right. \\
& \gamma_{\text {min }}=\left\{\begin{array}{ll}
\cos ^{-1}\left(\frac{V_{r r}}{V_{r \text { min }}}\right) & \text { if } V_{r r}<V_{r \text { min }}
\end{array} \mid \begin{array}{ll}
0 & \text { if } V_{r r} \geq V_{r \text { min }}
\end{array}\right.
\end{aligned}
$$

An average value of the angle $(\gamma)$ may be used to evaluate the central axis the T.D.Z .

$$
\gamma_{a v}=\frac{\gamma_{\max }+\gamma_{\min }}{2}
$$

The angle $\gamma_{\text {av }}$ represents the direction of the R.V between the missile and the target. The relative speed $\left|\mathrm{V}_{\mathrm{r}}\right|$ between the missile and the target is given by :

$$
\left|\mathrm{v}_{\mathrm{av}}\right|=\frac{\mathrm{v}_{\mathrm{rr}}}{\cos \gamma_{\mathrm{av}}}
$$

The delay time that the target at distance $\rho$ and is moving with R.V $\mathrm{V}_{\mathrm{av}}$, see figure 12 , needs to pass over the central axis of the T.D.Z (point P) is calculated as follow :

$$
t=\frac{\sqrt{\left(x_{T}-x_{p}\right)^{2}+\left(y_{T}-y_{p}\right)^{2}}}{V_{a v}}-t_{\text {in }}
$$

where :

$$
\begin{aligned}
V_{a v} & =\frac{V_{r r}}{\cos \gamma_{a v}}=\frac{\lambda \mathrm{f}_{\mathrm{d}}}{2 \cos \gamma_{\mathrm{av}}} \\
x_{p} & =\frac{\rho\left(\sin \phi-m_{1} \cos \phi\right)}{m_{2}-m_{1}}
\end{aligned}
$$




$$
\begin{aligned}
& y_{p}=m_{2} \mathrm{x}_{\mathrm{p}} \\
& m_{1}=\tan \left(\phi-\gamma_{a v}\right) \\
& m_{2}=\frac{V_{d}+V_{a v} \sin \left(\phi-\gamma_{a v}\right)}{V_{a v} \cos \left(\phi-\gamma_{a v}\right)} \\
& f_{d} \ldots \text { measured value on-board of the Doppler frequency } \\
& t_{\text {in }} \ldots \text { time of fuze inertia } \\
& \mathrm{V}_{d} \ldots \text { fragment speed } \\
& \phi \quad \ldots \text { antenna beam tilting angle }
\end{aligned}
$$

As a conclusion, from the measured value of the Doppler frequency the radial component $\left(V_{r r}\right)$ of the R.V is calculated. From the minimum and maximum expected values of R.V the minimum and maximum values of the angle $(\gamma)$ are calculated. An average value of $(\gamma)$ is considered and used to calculate the average value of the R.V $\left(V_{a v}\right)$. Then the delay time between the target detection and W.H initiation is calculated.

\section{4- CONCLUSION}

The ability of dynamic determination of M/T encounter conditions on-board of missile results in appreciable improvement of fuze performance. A factor for performance improvement (P.I.F) is defined as :

where

$$
\text { P. I. F }=\frac{\mathrm{Wi}^{-} \mathrm{W}_{0}}{\mathrm{~W}_{0}} * 100 \%
$$

$$
\begin{array}{ll}
\mathrm{W}_{\mathrm{i}} \ldots & \text { Improved performance } \\
\mathrm{W}_{\mathrm{o}} \ldots & \text { Normal performance }
\end{array}
$$

The improvement methods elaborated in the paper provides the following :

1-Improvement based on information about missile speed offers a P.I.F of $25.58 \%$. An appreciable improvement of such fuze performance is only expected in the case where the missile speed changes within a wide range and the target speed changes within a close range.

2-Improvement according to information about R.V modulus increases the P.I.F to be of $83.7 \%$. The performance of such R.F increases as the R.V modulus decreases.

3-If information about relative velocity vector could be available the probability of target destruction and the fuze performance would be maximum. 
The elaborated mathematical model, of performance improvement for R.F with variable A.B.P, is based only on information about the miss-distance $(\rho)$ and the elevation angle $(\phi)$ with respect to the coordinate system $(\rho, \theta, \phi)$ shown in figure 5 . As a result, the calculation procedure for matching between the F.A.Z and the T.D.Z is simplified and the tracker design becomes easier. Fortunately, information about $\theta$ is of a little importance due to the uniform distribution of the W.H fragments in the plane at which the angle $\theta$ is measured. A numerical differentiation method is proposed which enables to calculate, on-board of the missile, the instantaneous derivatives of $\rho$ and $\phi$ The measured values of $\rho$ and $\phi$ and their calculated derivatives are used to estimate the planer value of the R.V vector in the plane of fire. This estimated value determines the T.D.Z and the proper time of W.H initiation.

For R.F with variable delay time a mathematical model has been elaborated for performance improvement. In this model, the measured Doppler frequency and a pre-determined range of target velocity are used to estimate an average planer R.V vector. This estimated average value defines the T.D.Z and the delay time at which the W.H is initiated.

The results have proved that the R.F with variable A.B.P has better performance than that with variable delay time. for future work :

Referring to the functional diagram shown below, the following research points are available

- Design and implementation of R.F with controlled antenna beam pattern

- Sensors for on-board determination of target parameters

-The specification and selection of a microcomputer (processor, memory, interface) capable of real-time processing of algorithms for performance improvement and optimum initiation of the W.H .

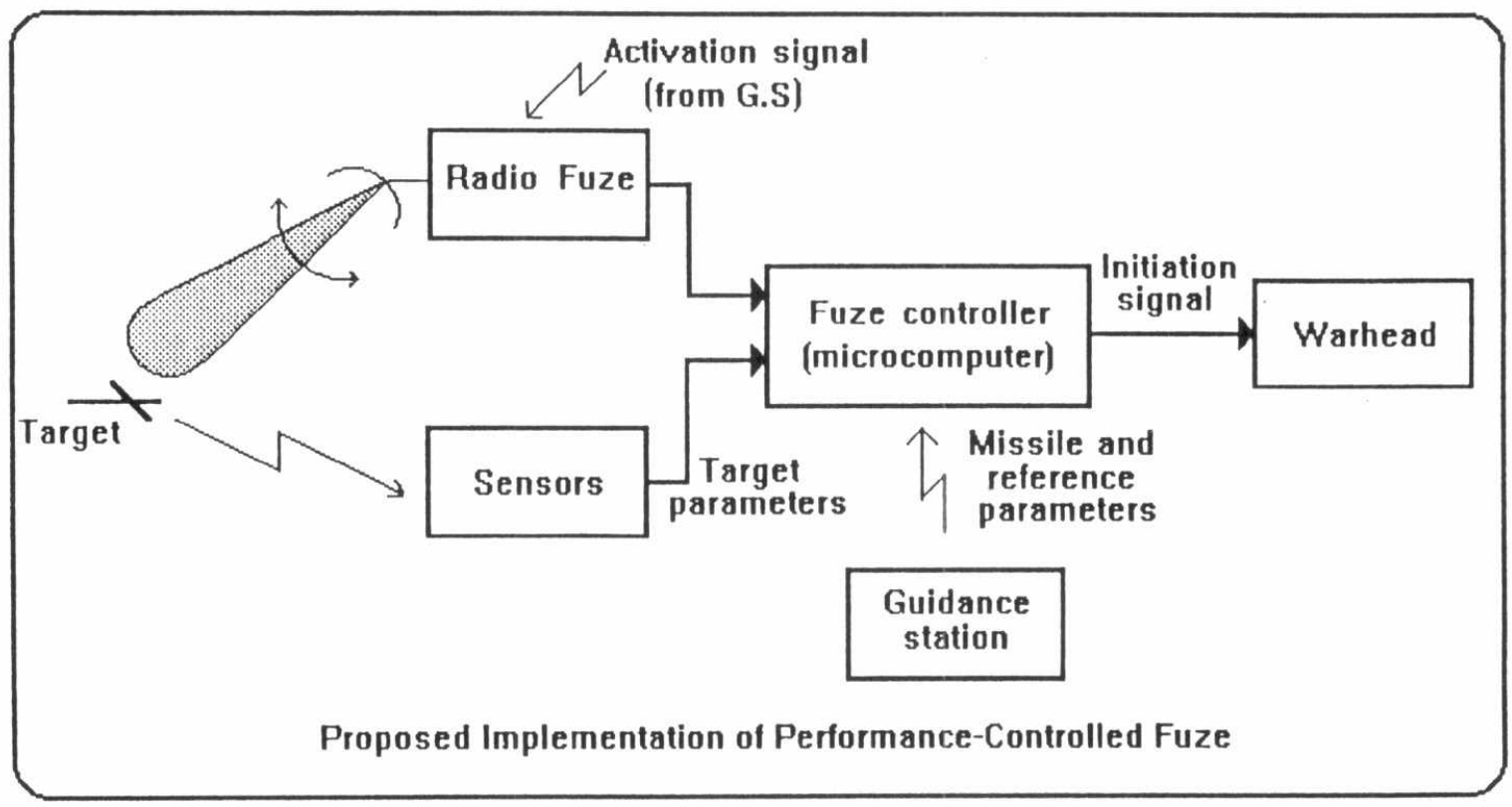




\section{5- REFERENCES}

[1]Otakar Dvořak,"Theory of radio fuzes for anti-aircraft rockets" Printed lecture 756, M.T.C,1967 [2]A.A Saleh ,"Optimization of fuzing action for proximity fuzes of AA/C guided missile" Thesis for M.Sc , M.T.C , 1995.

[3] Bp. Demidovich, I.A. Maron "Computational mathematics" Mir publisher, 1981.

[4] Merrill I. skolnik, "Introdution to radar system" McGraw-hill,1988.

[5] Dusek Josef, "radio fuze of anti-aircraft guided rocket taking advantage of Doppler effect" Printed lecture TSR 195, M.T.C,1968.

[6] Paul Zarchan, "Tactical and strategic missile guidance" Book publications AIAA, 1994.

[7] S.J. Parker, "Missile fuzing system" Technical report, March 1988.

[8] M.A. EL-Lithy "Testing device for Radio fuzes" Diploma project report , M.T.C ,1972. 

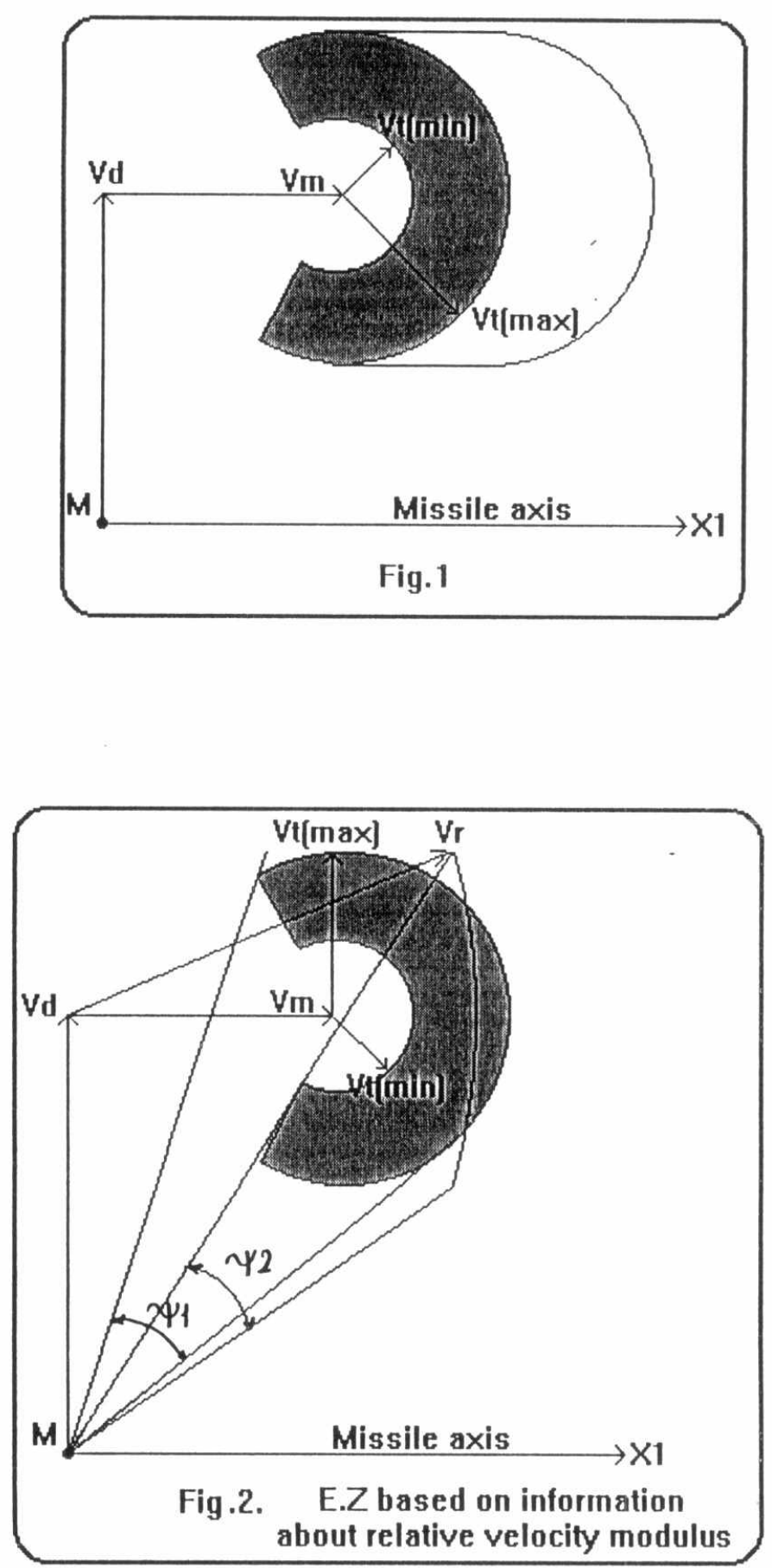

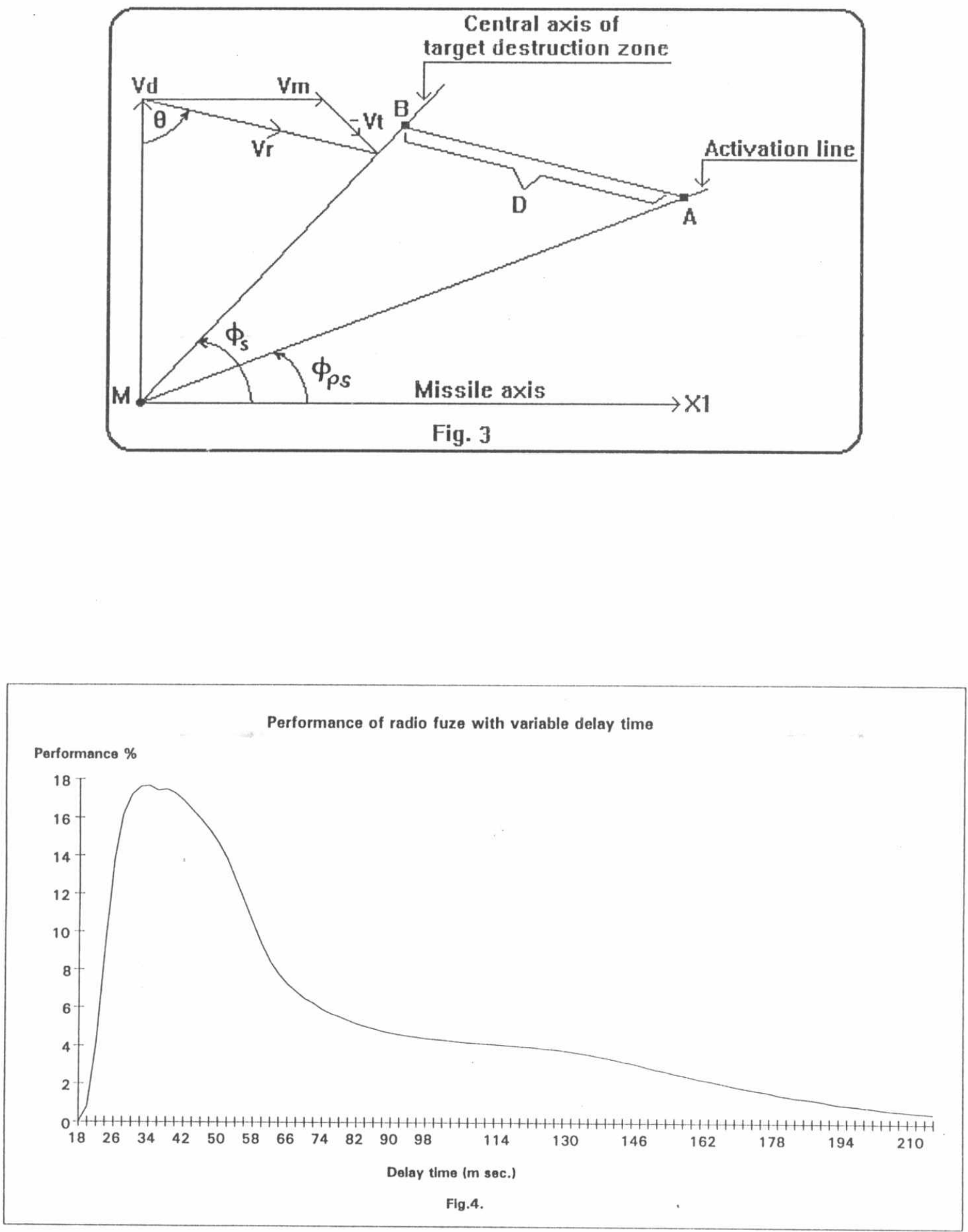

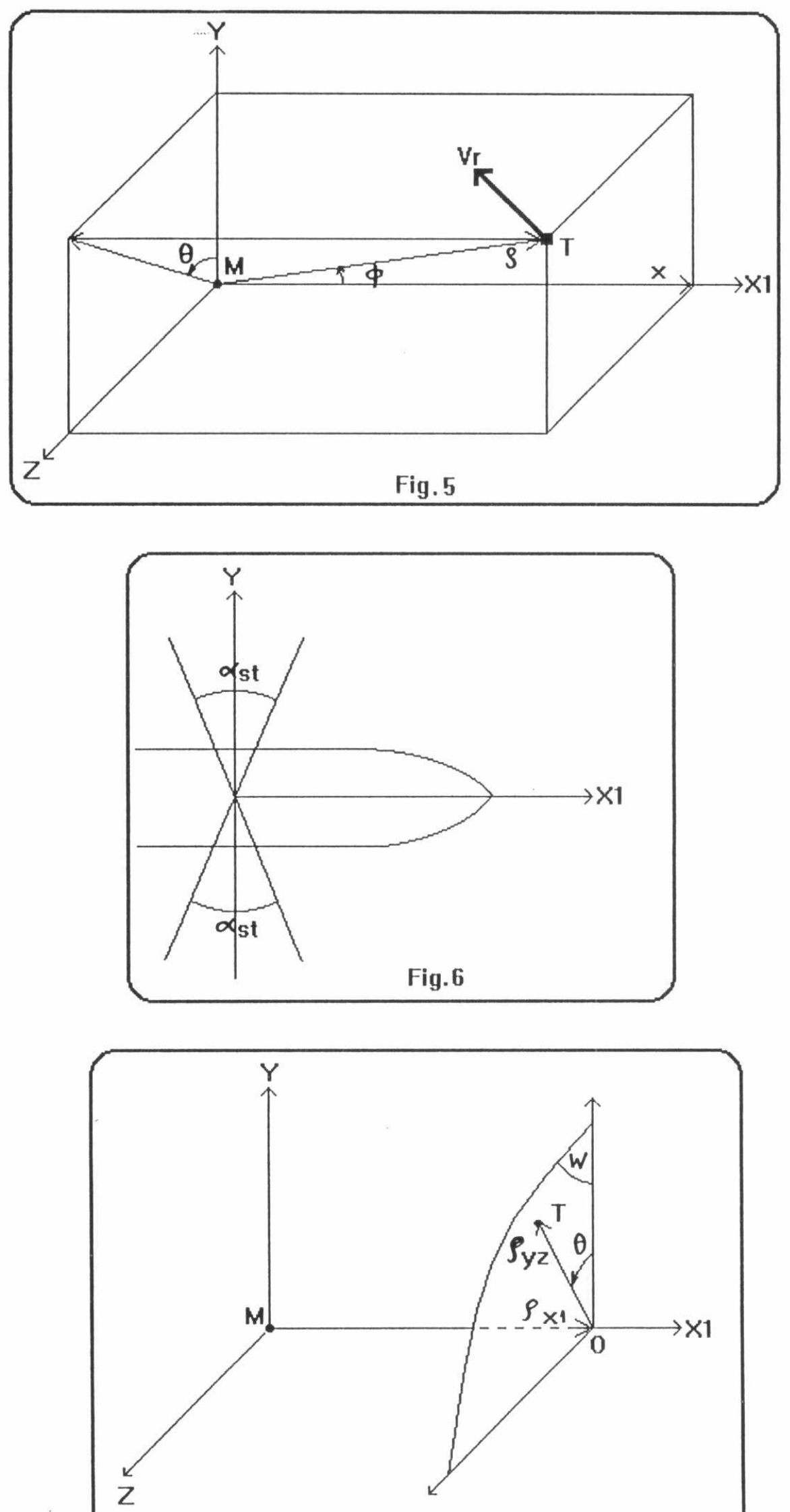

Fig. 7 

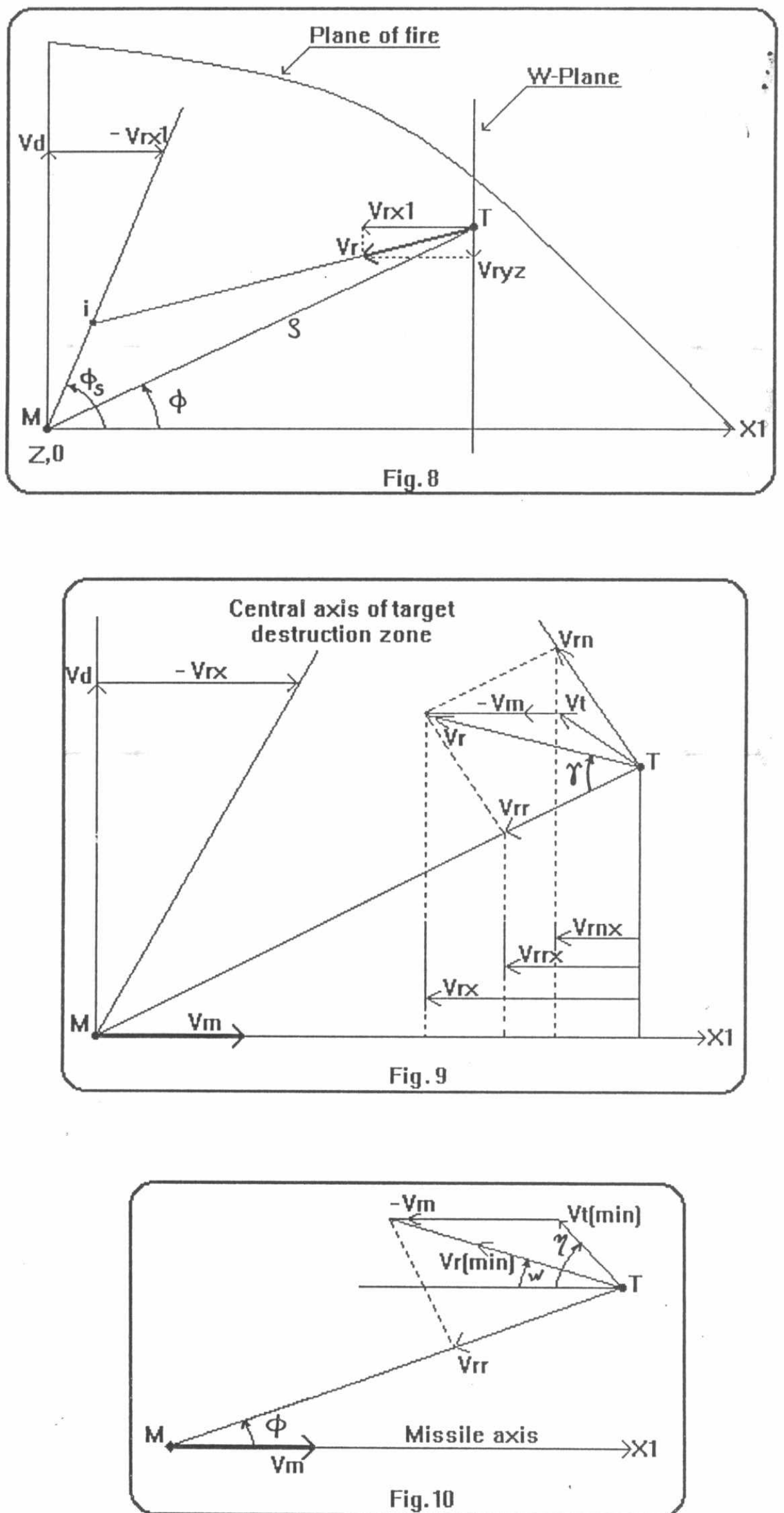

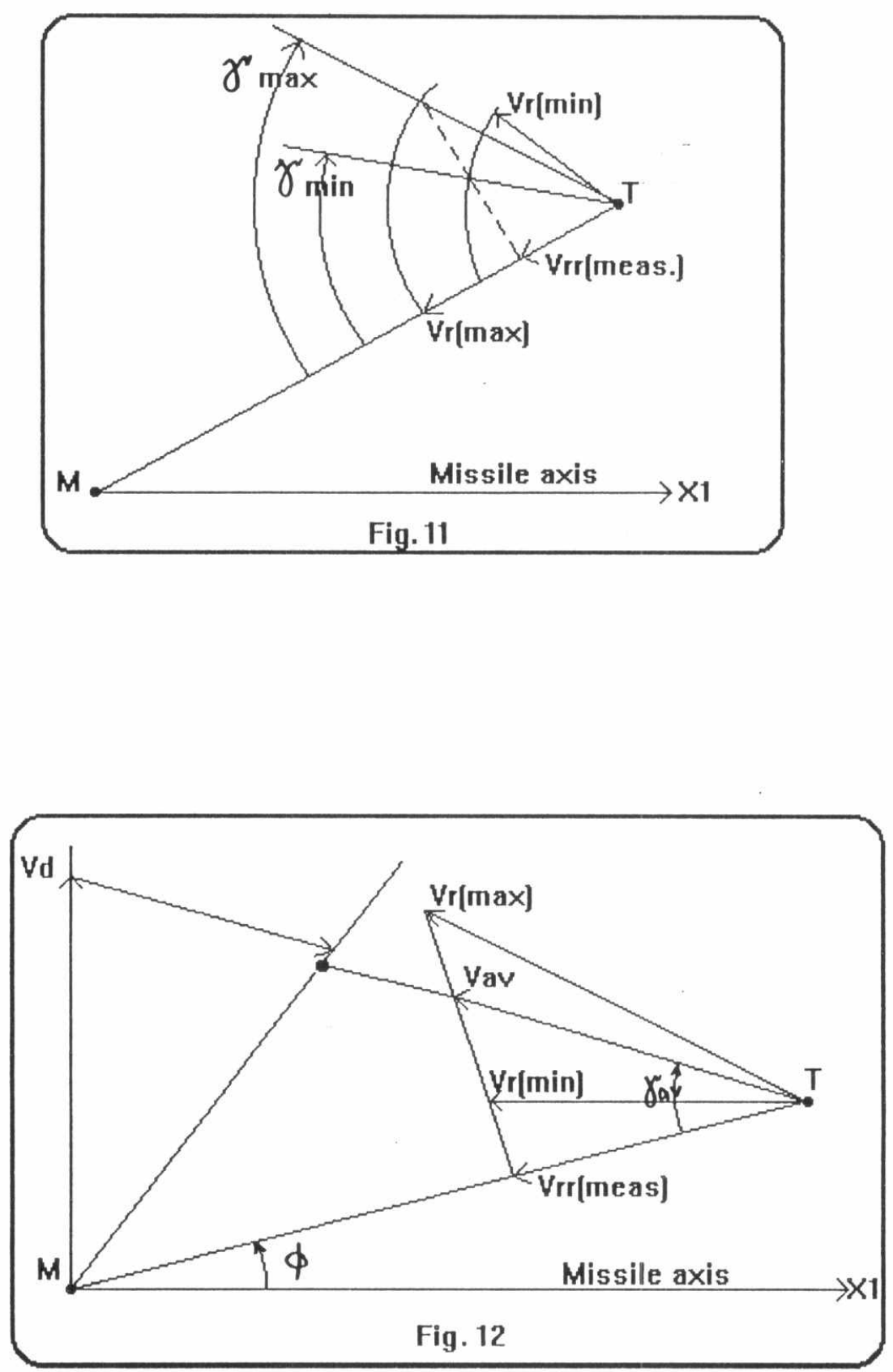